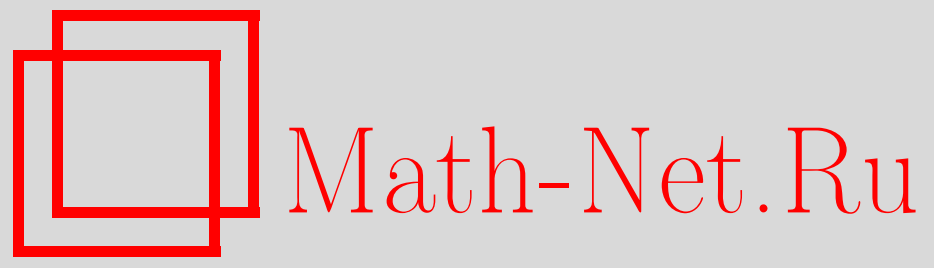

Ф. М. Малышев, Двойственность разностного и линейного методов в криптографии, Матем. вопр. криптогр., 2014, том 5, выпуск 3, 35-47

DOI: https://doi.org/10.4213/mvk128

Использование Общероссийского математического портала Math-Net.Ru подразумевает, что вы прочитали и согласны с пользовательским соглашением http://www . mathnet.ru/rus/agreement

Параметры загрузки:

IP : 54.162 .127 .20

26 апреля 2023 г., 18:25:01 
МАТЕМАТИЧЕСКИЕ ВОПРОСЫ КРИПТОГРАФИИ

2014 T. 5 № 3 C. 35-47

УДК 519.142.1

\title{
Двойственность разностного и линейного методов в криптографии
}

\author{
Ф. М. Малышев \\ Математический институт им. В.А. Стеклова РАН, Москва
}

Получено 22.IV.2013

Показано, что поиск разностных и вероятностных линейных соотношений для шифрпреобразований общего вида можно проводить с единых позиций. Приводятся разновидности схем Фейстеля и XSL-сетей, для каждой из которых построение разностных и линейных соотношений может производиться одной и той же последовательностью действий, необходимо только в одном случае в качестве исходных данных использовать таблицы локальных разностных характеристик, а в другом таблицы локальных линейных характеристик, все остальные исходные данные одинаковы.

Ключевые слова: разностный и линейный криптоанализ, шифрпреобразование, схемы Фейстеля, XSL-сети.

\section{The duality of differential and linear methods in cryptography}

\section{F. M. Malyshev}

Steklov Mathematical Institute of RAS, Moscow

Abstract. It is shown that the search for differential and linear probability relations for general type encryption transformations may be realized by the unified approach. We present examples of the construction of differential and linear relations for Feistel schemes and XSL-networks by the same sequence of operations, with inclusion in the input data tables of the local differential characteristics in the first case and tables of local linear characteristics in the second case.

Keywords: differential and linear cryptanalysis, encryption transformation, Feistel schemes, XSL- networks.

Citation: Mathematical Aspects of Cryptography, 2014, vol. 5, no. 3, pp. 35-47 (Russian)

(C) 2014 Ф. М. Малышев 
1. Введение. М. Матсуи в [3] обратил внимание на то, что для построения разностных и линейных соотношений криптосхемы DES можно пользоваться одним и тем же алгоритмом, нужно только в одном случае использовать разностные характеристики локальных нелинейных преобразований, а в другом - преобладания для локальных вероятностных линейных соотношений. В настоящей работе этому обстоятельству даётся объяснение в общем случае.

В п. 2 шифрпреобразование общего вида представляется функциональной схемой. В пп. 3 и 4 представлены соответственно разностный и линейный методы анализа, где прослеживается их двойственность. В пп. 5 и 6 приводятся самодвойственные схемы Фейстеля и XSL-сети.

Не умаляя значения первоисточников (см. [1], [2]), нам будет удобней в пп. 3 и 4 основываться на способах получения разностных и линейных соотношений, использованных в более поздних работах авторов алгоритма шифрования AES (см. [4], [5], [6]).

2. Функциональные схемы. Пусть $V_{N}=G F(2)^{N}$. Шифрпреобразование $F: V_{N} \rightarrow V_{M}, V_{N} \ni a \mapsto b=F(a) \in V_{M}$, задаём функциональной схемой, представляемой последовательностью выполняемых в определённом порядке линейных и нелинейных преобразований. Эту последовательность можно также рассматривать как схему компьютерной программы без циклов.

Пусть нелинейные преобразования $f_{i}: V_{n_{i}} \rightarrow V_{m_{i}}, \quad x_{i} \mapsto y_{i}=f_{i}\left(x_{i}\right)$, $i=1, \ldots, k$, в этой программе выполняются в указанном порядке. В промежутках между ними могут выполняться линейные преобразования. Через $x_{i} \in V_{n_{i}}$ обозначено значение аргумента отображения $f_{i}$, которое, в конечном счёте, выражается через $a \in V_{N}$ и является результатом выполнения некоторых предыдущих операций.

По отдельности линейные операции в шифрпреобразовании $F$ выписывать не будем. Ограничимся тем, что для некоторых матриц $c_{i j}$ размера $m_{i} \times n_{j}, i=0,1, \ldots, k, j=1, \ldots, k, k+1, \quad m_{0}=N, n_{k+1}=M$, можно записать $x_{j}=a c_{0 j}+\sum_{i=1}^{j-1} y_{i} c_{i j}, \quad j=1, \ldots, k, \quad b=a c_{0, k+1}+\sum_{i=1}^{k} y_{i} c_{i, k+1}$. Полагая $c_{i j}=0$ при $i \geq j$, из блоков $c_{i j}$ можно составить матрицу $C$ размера $\left(N+\sum_{i=1}^{k} m_{i}\right) \times\left(\sum_{i=1}^{k} n_{i}+M\right)$, для которой 


$$
\left(a, y_{1}, \ldots, y_{k}\right) C=\left(x_{1}, \ldots, x_{k}, b\right) .
$$

В матрице $C$ аккумулированы все линейные операции шифрпреобразования $F$.

Предлагаемое задание конкретного шифрпреобразования $F: V_{N} \rightarrow V_{M}$ не единственно. Можно, например, перейти к новым переменным $\tilde{a}=a S$, $\tilde{b}=b H, \quad \tilde{x}_{i}=x_{i} s_{i}, \quad \tilde{y}_{i}=y_{i} h_{i}, \quad i=1, \ldots, k$, где $S, H, s_{i}, h_{i}-$ невырожденные двоичные матрицы размеров, соответственно, $N, M, n_{i}, m_{i}$. Тогда $\tilde{f}_{i}(z)=f_{i}\left(z s_{i}^{-1}\right) h_{i}, z \in V_{n_{i}}$, и

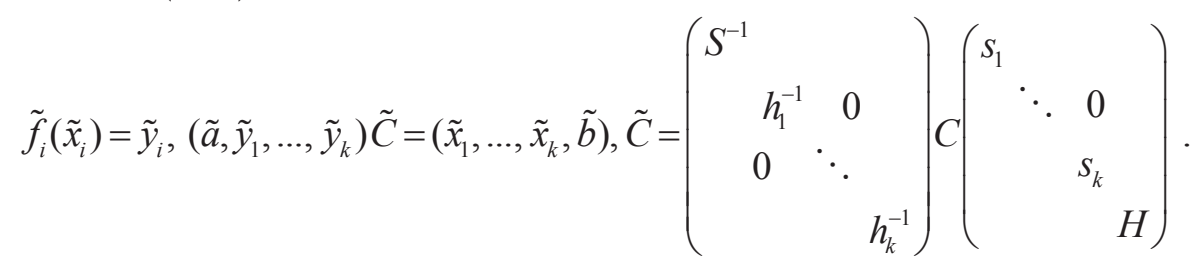

Задания $\left(C, f_{1}, \ldots, f_{k}\right)$ и $\left(\tilde{C}, \tilde{f}_{1}, \ldots, \tilde{f}_{k}\right)$ для шифрпреобразования $F$ далее считаем эквивалентными.

3. Разностный метод. Рассматривая равенства (1) для двух входов функциональной схемы $a^{(1)}, a^{(2)} \in V_{N}$ и вычитая (в поле $G F(2)$ ) одно из другого, получим

$$
\left(D^{\prime}, d_{1}^{\prime \prime}, \ldots, d_{k}^{\prime \prime}\right) C=\left(d_{1}^{\prime}, \ldots, d_{k}^{\prime}, D^{\prime \prime}\right),
$$

где $D^{\prime}=a^{(1)}+a^{(2)}, D^{\prime \prime}=b^{(1)}+b^{(2)}, d_{i}^{\prime \prime}=y_{i}^{(1)}+y_{i}^{(2)}, d_{i}^{\prime}=x_{i}^{(1)}+x_{i}^{(2)}, i=1, \ldots, k$. Здесь $b^{(1)}=F\left(a^{(1)}\right), b^{(2)}=F\left(a^{(2)}\right), x_{i}^{(1)}, x_{i}^{(2)}$ - значения аргументов $f_{i}$, когда входами всей функциональной схемы являются, соответственно, $a^{(1)}$ и $a^{(2)}$, $y_{i}^{(1)}=f_{i}\left(x_{i}^{(1)}\right), y_{i}^{(2)}=f_{i}\left(x_{i}^{(2)}\right), i=1, \ldots, k$.

Первоочередной задачей разностного метода является построение такой пары векторов $D^{\prime} \in V_{N}, D^{\prime \prime} \in V_{M}$, для которой импликации $a^{(1)}+a^{(2)}=D^{\prime} \Rightarrow b^{(1)}+b^{(2)}=D^{\prime \prime}$ отвечает как можно большая вероятность $p_{D^{\prime}, D^{\prime \prime}}=p_{D^{\prime}, D^{\prime \prime}}^{F}=\mathbf{P}\left\{F\left(a^{(1)}\right)+F\left(a^{(1)}+D^{\prime}\right)=D^{\prime \prime}\right\}$, вычисляемая по равновероятному распределению $a^{(1)}$ на $V_{N}$. Эта вероятность называется разностной характеристикой.

Пусть $D^{\prime} \in V_{N}, \quad D^{\prime \prime} \in V_{M}, \quad d_{i}^{\prime} \in V_{n_{i}}, \quad d_{i}^{\prime \prime} \in V_{m_{i}}, i=1, \ldots, k,-$ произвольный набор векторов, удовлетворяющий условию (3). Тогда импликация 
$a^{(1)}+a^{(2)}=D^{\prime} \Rightarrow b^{(1)}+b^{(2)}=D^{\prime \prime} \quad$ будет $\quad$ следовать из импликаций $x_{i}^{(1)}+x_{i}^{(2)}=d_{i}^{\prime} \Rightarrow y_{i}^{(1)}+y_{i}^{(2)}=d_{i}^{\prime \prime}, i=1, \ldots, k$. Это верно, поскольку матрица $C$ является блочно верхне треугольной. В самом деле, постоянно прибегая к равенству (3), последовательно убеждаемся в справедливости равенств:

$$
\begin{gathered}
a^{(1)}+a^{(2)}=D^{\prime} \Rightarrow x_{1}^{(1)}+x_{1}^{(2)}=d_{1}^{\prime} \Rightarrow y_{1}^{(1)}+y_{1}^{(2)}=d_{1}^{\prime \prime} \Rightarrow x_{2}^{(1)}+x_{2}^{(2)}=d_{2}^{\prime} \Rightarrow \\
\Rightarrow y_{2}^{(1)}+y_{2}^{(2)}=d_{2}^{\prime \prime} \Rightarrow \ldots \Rightarrow x_{k}^{(1)}+x_{k}^{(2)}=d_{k}^{\prime} \Rightarrow y_{k}^{(1)}+y_{k}^{(2)}=d_{k}^{\prime \prime} \Rightarrow b^{(1)}+b^{(2)}=D^{\prime \prime} .
\end{gathered}
$$

При наличии набора разностей $D=\left(\left(d_{i}^{\prime}, d_{i}^{\prime \prime}\right), i=1, \ldots, k\right), \quad d_{i}^{\prime} \in V_{n_{i}}$, $d_{i}^{\prime \prime} \in V_{m_{i}}$, удовлетворяющего условию (3) при некоторых краевых значениях $D^{\prime} \in V_{N}, \quad D^{\prime \prime} \in V_{M}, \quad$ в качестве приближения для вероятности $p_{D^{\prime}, D^{\prime \prime}}=\mathbf{P}\left\{F\left(a^{(1)}\right)+F\left(a^{(1)}+D^{\prime}\right)=D^{\prime \prime}\right\}$, характеризующей разностное соотношение $\left(D^{\prime}, D^{\prime \prime}\right) \in V_{N} \times V_{M}$ и вычисляемой при равновероятном $a^{(1)} \in V_{N}$, рассматривают произведение $\tilde{p}_{\mathrm{D}}=\prod_{i=1}^{k} p_{d_{i}^{\prime}, d_{i}^{\prime \prime}}^{f_{i}}$, в котором каждый сомножитель $p_{d_{i}^{\prime}, d_{i}^{\prime \prime}}^{f_{i}}=\mathbf{P}\left\{f_{i}\left(x_{i}^{(1)}\right)+f_{i}\left(x_{i}^{(1)}+d_{i}^{\prime}\right)=d_{i}^{\prime \prime}\right\}$ вычисляется по равновероятному распределению $x_{i}^{(1)}$ на $V_{n_{i}}$.

Использование приближения $\tilde{p}_{\mathrm{D}}$ для $p_{D^{\prime}, D^{n}}$ оправдывается следующим рассуждением. Если $a^{(2)}=a^{(1)}+D^{\prime}$, а равновероятность $a^{(1)} \in V_{N}$ обеспечивает равновероятность $x_{i}^{(1)} \in V_{n_{i}}$ для всех $i=1, \ldots, k$, и если события $\left\{y_{i}^{(1)}+y_{i}^{(2)}=d_{i}^{\prime \prime}\right\}, \quad i=1, \ldots, k, \quad$ независимы в совокупности, то согласно (3)

$$
\begin{gathered}
p_{D^{\prime}, D^{\prime \prime}} \geq \mathbf{P}\left\{y_{i}^{(1)}+y_{i}^{(2)}=d_{i}^{\prime \prime}, i=1, \ldots, k\right\}=\prod_{i=1}^{k} \mathbf{P}\left\{y_{i}^{(1)}+y_{i}^{(2)}=d_{i}^{\prime \prime}\right\}= \\
=\prod_{i=1}^{k} \mathbf{P}\left\{f_{i}\left(x_{i}^{(1)}\right)+f_{i}\left(x_{i}^{(2)}\right)=d_{i}^{\prime \prime}\right\}=\prod_{i=1}^{k} \mathbf{P}\left\{f_{i}\left(x_{i}^{(1)}\right)+f_{i}\left(x_{i}^{(1)}+d_{i}^{\prime}\right)=d_{i}^{\prime \prime}\right\}=\tilde{p}_{\mathbf{D}} .
\end{gathered}
$$

Выбор наборов $\boldsymbol{D}=\left(\left(d_{i}^{\prime}, d_{i}^{\prime \prime}\right), i=1, \ldots, k\right)$, удовлетворяющих условию (3) при некоторых $D^{\prime} \in V_{N} \backslash\{0\}, D^{\prime \prime} \in V_{M}$, нацелен на увеличение $\tilde{p}_{\mathrm{D}}$. Если $d_{i}^{\prime}=0, d_{i}^{\prime \prime} \neq 0$ или $d_{i}^{\prime} \neq 0, d_{i}^{\prime \prime}=0$ при некотором $i \in\{1, \ldots, k\}$, а $f_{i}$ биективно, то $\tilde{p}_{\mathrm{D}}=0$. Естественно, что наборы $\boldsymbol{D}$ с такими “дефектами" не рассматривают. Наличие $i \in\{1, \ldots, k\}$ с $d_{i}^{\prime}=0$ и $d_{i}^{\prime \prime}=0$, напротив, является желательным, тогда $p_{0,0}^{f_{i}}=1$. При поиске разностных соотношений $\left(D^{\prime}, D^{\prime \prime}\right), D^{\prime} \neq 0$, стараются увеличивать число таких номеров $i$, 
называемых благоприятными (не допуская при этом, чтобы равенства $d_{i}^{\prime}=0, d_{i}^{\prime \prime}=0$ выполнялись при всех $\left.i \in\{1, \ldots, k\}\right)$.

Если применять разностный метод для двух эквивалентных заданий шифрпреобразования $F$, для $\left(C, f_{1}, \ldots, f_{k}\right)$ и $\left(\tilde{C}, \tilde{f}_{1}, \ldots, \tilde{f}_{k}\right)$, то в силу равенств (2) будем получать одинаковый результат. В частности, максимально возможное число благоприятных номеров $i$ для эквивалентных заданий будет одинаковое. Выше сказанное следует из того, что при $\tilde{D}^{\prime}=D^{\prime} S, \quad \tilde{D}^{\prime \prime}=D^{\prime \prime} H, \quad \tilde{d}_{i}^{\prime}=d_{i}^{\prime} s_{i}, \quad \tilde{d}_{i}^{\prime \prime}=d_{i}^{\prime \prime} h_{i}, \quad i=1, \ldots, k$, справедливы равенства $p_{d_{i}^{\prime}, d_{i}^{\prime \prime}}^{f_{f^{\prime}}}=p_{\tilde{d}_{i}^{\prime}, \tilde{d}_{i}^{\prime \prime}}^{\tilde{f}_{i}}$, а равенство (3) равносильно равенству $\left(\tilde{D}^{\prime}, \tilde{d}_{1}^{\prime \prime}, \ldots, \tilde{d}_{k}^{\prime \prime}\right) \tilde{C}=\left(\tilde{d}_{1}^{\prime}, \ldots, \tilde{d}_{k}^{\prime}, \tilde{D}^{\prime \prime}\right)$.

4. Линейный метод. Первоочередной задачей линейного метода является построение такой пары вектор-столбцов $L^{\prime} \in V_{N}^{*}, L^{\prime \prime} \in V_{M}^{*} \backslash\{0\}$, для которой модуль преобладания $\delta_{L^{\prime}, L^{\prime \prime}}=\delta_{L^{\prime}, L^{\prime \prime}}^{F}=2 \mathbf{P}\left\{a L^{\prime}=b L^{\prime \prime}\right\}-1$ имеет как можно большее значение; здесь $b=F(a)$, а $a \in V_{N}$ имеет равномерное распределение на $V_{N}$. Элементы векторного пространства $V_{K}=G F(2)^{K}$, $K \in \mathbb{N}$, представляем в виде строк, элементы множества линейных функций на $V_{K}$, обозначаемого через $V_{K}^{*}$, представляем вектор-столбцами из $K$ элементов поля $G F(2)$.

Парой $\left(L^{\prime}, L^{\prime \prime}\right) \in V_{N}^{*} \times\left(V_{M}^{*} \backslash\{0\}\right)$ задаётся линейное вероятностное соотношение, характеризуемое преобладанием $\delta_{L^{\prime}, L^{\prime \prime}}$ и записываемое в виде $a L^{\prime} \simeq b L^{\prime \prime}$ или $a L^{\prime}+b L^{\prime \prime} \simeq 0$. Качественно последние выражения можно воспринимать как приближённые равенства, фактически же они являются обозначением условия $\mathbf{P}\left\{a L^{\prime}=b L^{\prime \prime}\right\}=\frac{1+\delta_{L^{\prime}, L^{\prime \prime}}}{2}$.

В предыдущем пункте по локальным разностным соотношениям $\left(d_{i}^{\prime}, d_{i}^{\prime \prime}\right), i=1, \ldots, k$, удовлетворяющим условию (3), получали глобальное разностное соотношение $\left(D^{\prime}, D^{\prime \prime}\right) \in\left(V_{N} \backslash\{0\}\right) \times V_{M}$.

Поступая в некотором смысле аналогично, вероятностные линейные соотношения $a L^{\prime}+b L^{\prime \prime} \simeq 0$, будем получать формальным сложением всех локальных вероятностных линейных соотношений $x_{i} l_{i}^{\prime}+y_{i} l_{i}^{\prime \prime} \simeq 0, l_{i}^{\prime} \in V_{n_{i}}^{*}$, $l_{i}^{\prime \prime} \in V_{m_{i}}^{*}, \quad y_{i}=f_{i}\left(x_{i}\right), \quad i=1, \ldots, k, \quad$ характеризующихся преобладаниями 
$\delta_{i}=\delta_{l_{i}, l_{i}^{\prime}}^{f_{i}}=2 \mathbf{P}\left\{x_{i} l_{i}^{\prime}=y_{i} l_{i}^{\prime \prime}\right\}-1$, которые вычисляются в предположении о равномерности распределения $x_{i}$ на $V_{n_{i}}$.

В качестве приближения для $\delta_{L^{\prime}, L^{\prime \prime}}$ рассматривают $\tilde{\delta}=\delta_{1} \cdot \ldots \cdot \delta_{k}$. Если равновероятность $a \in V_{N}$ обеспечивает равновероятность $x_{i} \in V_{n_{i}}$ для всех $i=1, \ldots, k$, и булевы случайные величины $x_{i} l_{i}^{\prime}+f_{i}\left(x_{i}\right) l_{i}^{\prime \prime}, \quad i=1, \ldots, k$, независимы в совокупности, то $\delta_{L^{\prime}, L^{\prime \prime}}=\tilde{\delta}$. Это и оправдывает рассмотрение $\tilde{\delta}$ как приближения к преобладанию $\delta_{L^{\prime}, L^{\prime \prime}}$.

Пусть далее $x=\left(x_{1}, \ldots, x_{k}\right), y=\left(y_{1}, \ldots, y_{k}\right), l^{\prime}=\left(\begin{array}{l}l_{1}^{\prime} \\ \vdots \\ l_{k}^{\prime}\end{array}\right), l^{\prime \prime}=\left(\begin{array}{l}l_{1}^{\prime \prime} \\ \vdots \\ l_{k}^{\prime \prime}\end{array}\right)$. В результате означенных сложений локальных вероятностных линейных соотношений все промежуточные переменные $x_{i}, y_{i}$ должны сократиться за счёт соотношений (1). В конечном счёте сумма должна быть выражена через компоненты векторов $a \in V_{N}$ и $b \in V_{M}$. Другими словами, набор $\mathfrak{L}=\left(\left(l_{i}^{\prime}, l_{i}^{\prime \prime}\right), i=1, \ldots, k\right)$ должен быть таким, чтобы при некоторых $L^{\prime} \in V_{N}^{*}$ и $L^{\prime \prime} \in V_{M}^{*}$ имело место тождество

$$
\begin{aligned}
& 0=\sum_{i=1}^{k}\left(x_{i} l_{i}^{\prime}+y_{i} l_{i}^{\prime \prime}\right)+a L^{\prime}+b L^{\prime \prime}=x l^{\prime}+y l^{\prime \prime}+a L^{\prime}+b L^{\prime \prime}= \\
& =(a, y)\left(\begin{array}{l}
L^{\prime} \\
l^{\prime \prime}
\end{array}\right)+(x, b)\left(\begin{array}{l}
l^{\prime} \\
L^{\prime \prime}
\end{array}\right)=(a, y)\left(\begin{array}{l}
L^{\prime} \\
l^{\prime \prime}
\end{array}\right)+(a, y) C\left(\begin{array}{c}
l^{\prime} \\
L^{\prime \prime}
\end{array}\right)=(a, y)\left[\left(\begin{array}{c}
L^{\prime} \\
l^{\prime \prime}
\end{array}\right)+C\left(\begin{array}{c}
l^{\prime} \\
L^{\prime \prime}
\end{array}\right)\right] \text {. }
\end{aligned}
$$

Это означает, что аналогом соотношения (3) здесь выступает равенство

$$
C\left(\begin{array}{c}
l^{\prime} \\
L^{\prime \prime}
\end{array}\right)=\left(\begin{array}{c}
L^{\prime} \\
l^{\prime \prime}
\end{array}\right)
$$

или, после транспонирования, $\left(l_{1}^{\prime T}, \ldots, l_{k}^{\prime T}, L^{\prime \prime T}\right) C^{T}=\left(L^{\prime T}, l_{1}^{\prime \prime T}, \ldots, l_{k}^{\prime T}\right)$. Наконец, окончательно имеем:

$$
\left(L^{\prime \prime T}, l_{k}^{\prime T}, \ldots, l_{1}^{\prime T}\right) C^{*}=\left(l_{k}^{\prime \prime T}, \ldots, l_{1}^{\prime \prime T}, L^{\prime T}\right),
$$

где матрица $C^{*}=\left\|c_{i j}^{*}\right\|, i=0,1, \ldots, k, j=1, \ldots, k, k+1$, получается из матрицы $C^{T}$ центрально-симметричной перестановкой блоков. В результате: $c_{i j}^{*}=c_{k+1-j, k+1-i}^{T}$, и $c_{i j}^{*}=0$ при $i \geq j$. 
Условие (4) совершенно аналогично условию (3), но с другой матрицей: с матрицей $C^{*}$, получающейся из $C$ в результате несложных преобразований. Как и в разностном методе, выбор наборов $\mathfrak{L}=\left(\left(l_{i}^{\prime}, l_{i}^{\prime \prime}\right), i=1, \ldots, k\right)$, удовлетворяющих условию (4) при некоторых $L^{\prime} \in V_{N}^{*}, \quad L^{\prime \prime} \in V_{M}^{*} \backslash\{0\}$, нацелен на увеличение модуля произведения $\tilde{\delta}_{\mathfrak{L}}=\delta_{1} \cdot \ldots \cdot \delta_{k}$. Если $l_{i}^{\prime} \neq 0, l_{i}^{\prime \prime}=0$ или $l_{i}^{\prime}=0, l_{i}^{\prime \prime} \neq 0$ при некотором $i \in\{1, \ldots, k\}$, а $f_{i}$ биективно, то $\tilde{\delta}_{\mathfrak{L}}=0$. Наличие $i \in\{1, \ldots, k\}$ с $l_{i}^{\prime}=0$ и $l_{i}^{\prime \prime}=0$, напротив, является желательным, тогда $\delta_{i}=1$. При поиске соотношений $a L^{\prime} \simeq b L^{\prime \prime}, \quad L^{\prime \prime} \neq 0$, стараются увеличивать число таких номеров $i$, называемых благоприятными (не допуская при этом, чтобы равенства $d_{i}^{\prime}=0, d_{i}^{\prime \prime}=0$ выполнялись при всех $\left.i \in\{1, \ldots, k\}\right)$.

Как и в разностном методе, для двух эквивалентных заданий $\left(C, f_{1}, \ldots, f_{k}\right)$ и $\left(\tilde{C}, \tilde{f}_{1}, \ldots, \tilde{f}_{k}\right)$ одного и того же шифрпреобразования $F$ результаты применения линейного метода в силу равенств (2) будут одинаковыми. В частности, максимально возможное число благоприятных номеров $i$ для эквивалентных заданий одинаковое. Оба этих замечания следуют из того, что при $\tilde{L}^{\prime}=S^{-1} L^{\prime}, \tilde{L}^{\prime \prime}=H^{-1} L^{\prime \prime}, \tilde{l}_{i}^{\prime}=s_{i}^{-1} l_{i}^{\prime}, \tilde{l}_{i}^{\prime \prime}=h_{i}^{-1} l_{i}^{\prime \prime}, i=1, \ldots, k$, справедливы равенства $\delta_{l_{i}^{\prime}, l_{i}^{\prime \prime}}^{f_{i}}=\delta_{\tilde{l}_{i}^{\prime}, \tilde{l}_{i}^{\prime \prime}}^{\tilde{f}_{i}}$, а равенство (4) равносильно равенству

$$
\left(\tilde{L}^{\prime \prime T}, \tilde{l}_{k}^{T}, \ldots, \tilde{l}_{1}^{\prime T}\right) \tilde{C}^{*}=\left(\tilde{l}_{k}^{\prime \prime T}, \ldots, \tilde{l}_{1}^{\prime \prime T}, \tilde{L}^{\prime T}\right) .
$$

С точки зрения нахождения соответственно разностных и линейных соотношений рассматриваемые два метода принципиально не различаются. Реализация линейного метода совпадает с реализацией разностного, нужно лишь вместо исходной матрицы $C$ использовать матрицу $C^{*}$, а вместо таблиц значений $\left[p_{d_{i}^{\prime}, d_{i}^{\prime \prime}}^{f_{i}}\right], d_{i}^{\prime} \in V_{n_{i}}, d_{i}^{\prime \prime} \in V_{m_{i}}, i=1, \ldots, k$, использовать таблицы значений $\left[\left|\delta_{l_{i}^{\prime}, l_{i}^{\prime \prime}}^{f_{i}}\right|\right], l_{i}^{\prime} \in V_{n_{i}}^{*}, l_{i}^{\prime \prime} \in V_{m_{i}}^{*}, i=1, \ldots, k$. Роль векторов $d_{i}^{\prime} \in V_{n_{i}}$, $d_{i}^{\prime \prime} \in V_{m_{i}}, \quad i=1, \ldots, k, \quad D^{\prime} \in V_{N}, \quad D^{\prime \prime} \in V_{M}$, в линейном методе выполняют, соответственно, $l_{k+1-i}^{\prime \prime T} \in V_{m_{k+1-i}}, l_{k+1-i}^{\prime T} \in V_{n_{k+1-i}}, L^{\prime \prime T} \in V_{M}, L^{\prime T} \in V_{N}$. Наконец, легко проверяемое равенство $\left(C^{*}\right)^{*}=C$ даёт все основания говорить о наличии двойственности между линейным и разностным методами.

Определение. Шифрпреобразование $F$, заданное функциональной схемой $\left(C, f_{1}, \ldots f_{k}\right)$, считаем самодвойственным, если $C^{*}=C$ или $C^{*}=\tilde{C}$ для некоторого эквивалентного задания $F$. 
В заключение настоящего пункта приведём дополнительные доводы, обосновывающие указанную двойственность. Разностный и линейный методы на этапе построения соответственно разностных и линейных соотношений по функциональному заданию $\left(C, f_{1}, \ldots, f_{k}\right)$ шифрпреобразования $F: V_{N} \rightarrow V_{M}$ непосредственно используют только матрицу $C$ в соотношениях (3) и (4). Вместо каждого отображения $f_{i}: V_{n_{i}} \rightarrow V_{m_{i}}$, $i=1, \ldots, k$, в этих методах рассматриваются две таблицы размера $2^{n_{i}} \times 2^{m_{i}}$ : $\left[p_{d^{\prime}, d^{\prime \prime}}^{f_{f^{\prime}}}\right]_{\left(d^{\prime}, d^{\prime \prime}\right) \in V_{n_{i}} \times V_{m_{i}}}$ и $\left[\left|\delta_{l^{\prime}, l^{\prime}}^{f_{i}}\right|\right]_{\left(l^{\prime}, l^{\prime \prime}\right) \in V_{n_{i}}^{*} \times V_{m_{i}}^{*}}$. По большей части приведённые выше для этих двух методов рассуждения применимы к абстрактно определяемой схеме

$$
\mathcal{F}=\left(C,\left[p_{d^{\prime}, d^{\prime \prime}}^{(i)}\right]_{\left(d^{\prime}, d^{\prime \prime}\right) \in V_{n_{i}} \times V_{m_{i}}},\left[\delta_{l^{\prime}, l^{\prime}}^{(i)}\right]_{\left(l^{\prime}, l^{\prime \prime}\right) \in V_{n_{i}}^{*} \times V_{m_{i}}^{*}}, i=1, \ldots, k\right),
$$

соответствующей последовательностям натуральных чисел $m_{0}=N$, $m_{1}, \ldots, m_{k}$ и $n_{1}, \ldots, n_{k}, n_{k+1}=M$. В схеме $\mathcal{F}$ элементами таблиц являются числа из отрезка $[0,1]$, а $C=\left\|c_{i j}\right\|, i=0,1, \ldots, k, j=1, \ldots, k, k+1,-$ блочная верхне-треугольная матрица над полем $G F(2)$, состоящая из блоков $c_{i j}$ размера $m_{i} \times n_{j}, c_{i j}=0$ при $i \geq j$.

Двойственная $\mathcal{F}$ схема, соответствующая последовательностям $M, n_{k}, \ldots, n_{1}$ и $m_{k}, \ldots, m_{1}, N$, определяется следующим образом:

$$
\mathcal{F}^{*}=\left(C^{*},\left[\hat{p}_{d^{\prime}, d^{\prime \prime}}^{(i)}\right]_{\left(d^{\prime}, d^{\prime \prime}\right) \in V_{m_{k+1-i}} \times V_{n_{k+1-i}}},\left[\hat{\delta}_{l^{\prime}, l^{\prime}}^{(i)}\right]_{\left(l^{\prime}, l^{\prime \prime}\right) \in V_{m_{k+1-i}}^{*} \times V_{n_{k+1-i}}^{*}}, i=1, \ldots, k\right),
$$

$\hat{p}_{d^{\prime}, d^{\prime \prime}}^{(i)}=\delta_{d^{r T}, d^{\prime T}}^{(k+1-i)}, \quad d^{\prime} \in V_{m_{k+1-i}}, \quad d^{\prime \prime} \in V_{n_{k+1-i}}, \quad \hat{\delta}_{l^{\prime}, l^{\prime \prime}}^{(i)}=p_{l^{r T}, l^{T^{T}}}^{(k+1)}, \quad l^{\prime} \in V_{m_{k+1-i}}^{*}, \quad l^{\prime \prime} \in V_{n_{k+1-i}}^{*}$, $i=1, \ldots, k$.

При наличии задания $\left(C, f_{1}, \ldots, f_{k}\right)$ шифрпреобразования $F$ схема $\mathcal{F}$ возникает естественным образом, но далеко не любая схема вида (5) так реализуется. Более того, какая-то схема $\mathcal{F}$ может отвечать заданию $\left(C, f_{1}, \ldots, f_{k}\right)$ некоторого шифрпреобразования $F$, но для двойственной ей схемы $\mathcal{F}^{*}$ такого задания не будет.

Выявленная двойственность между линейным и разностным методами означает, что применение линейного метода к схеме $\mathcal{F}$ отвечает применению разностного метода к схеме $\mathcal{F}^{*}$, и наоборот, применение разностного метода к схеме $\mathcal{F}$ отвечает применению линейного метода к 
схеме $\mathcal{F}^{*}$. Так как $\left(\mathcal{F}^{*}\right)^{*}=\mathcal{F}$, то в приведённых замечаниях шифрпреобразования $\mathcal{F}$ и $\mathcal{F}^{*}$ можно поменять местами.

5. Самодвойственные схемы Фейстеля. Если $C^{*}=C$, то для разностного и линейного методов совпадают основные условия (3) и (4), налагаемые, соответственно, на локальные разностные соотношения и локальные линейные соотношения. В настоящем пункте подобные ситуации выявляются для схем Фейстеля.

В большей части современных реализаций схемы Фейстеля блок $a=\left(B_{0}, B_{1}\right) \in V_{n} \times V_{n}$ открытого текста преобразуется за $r$ итераций в блок $b=\left(B_{r+1}, B_{r}\right) \in V_{n} \times V_{n}$ шифрованного текста в соответствии с равенствами

$$
B_{i+1}=\Pi_{i}\left(B_{i}\right) \cdot \Lambda+B_{i-1}, i=1, \ldots, r,
$$

где $\Lambda \in G L(n, 2)$ - невырожденная двоичная матрица размера $n=m k$, $\Pi_{i}: V_{n} \rightarrow V_{n}-$ подстановка, $\Pi_{i}=\left(\pi_{i 1}, \ldots, \pi_{i k}\right), \pi_{i j}: V_{m} \rightarrow V_{m}, j=1, \ldots, k,-$ локальные подстановки, зависящие от итерационных ключей, $V_{n}=\left(V_{m}\right)^{k}$.

Полагая вначале в качестве нелинейных преобразований не подстановки $\pi_{i j}, i=1, \ldots, r, j=1, \ldots, k$, а подстановки $\Pi_{i}, i=1, \ldots, r$, будем иметь: $x_{i}=B_{i}, y_{i}=\Pi_{i}\left(x_{i}\right)$. Матрица $C$, аккумулирующая в себе все линейные операции схемы Фейстеля, имеет размер $(r+2) n \times(r+2) n$ и принимает (для определённости - при чётном $r$ ) представленный на рис. 1 вид.

Через $I$ на рис. 1 обозначена единичная матрица размера $n \times n$, а через 0 - нулевая матрица размера $n \times n$.

\begin{tabular}{|c|c|c|c|c|c|c|c|c|}
\hline 0 & $\mathrm{I}$ & 0 & $\ldots$ & $\mathrm{I}$ & 0 & $\mathrm{I}$ & 0 & $\mathrm{I}$ \\
\hline $\mathrm{I}$ & 0 & $\mathrm{I}$ & $\ldots$ & 0 & $\mathrm{I}$ & 0 & $\mathrm{I}$ & 0 \\
\hline 0 & $\Lambda$ & 0 & $\ldots$ & $\Lambda$ & 0 & $\Lambda$ & 0 & $\Lambda$ \\
\hline 0 & 0 & $\Lambda$ & $\ldots$ & 0 & $\Lambda$ & 0 & $\Lambda$ & 0 \\
\hline 0 & 0 & 0 & $\ldots$ & $\Lambda$ & 0 & $\Lambda$ & 0 & $\Lambda$ \\
\hline$\vdots$ & $\vdots$ & $\vdots$ & $\ldots$ & $\vdots$ & $\vdots$ & $\vdots$ & $\vdots$ & $\vdots$ \\
\hline 0 & 0 & 0 & $\ldots$ & 0 & $\Lambda$ & 0 & $\Lambda$ & 0 \\
\hline 0 & 0 & 0 & $\ldots$ & 0 & 0 & $\Lambda$ & 0 & $\Lambda$ \\
\hline 0 & 0 & 0 & $\ldots$ & 0 & 0 & 0 & $\Lambda$ & 0 \\
\hline
\end{tabular}

Рис. 1 
В качестве нелинейных (“далее неделимых”) преобразований в схеме Фейстеля рассматривают не подстановки $\Pi_{i}: V_{n} \rightarrow V_{n}$, а локальные подстановки $\pi_{i j}: V_{m} \rightarrow V_{m}, i=1, \ldots, r, j=1, \ldots, k$. Но от этого вид матриц $C$ и $C^{*}$ не изменяется. Нужно только матрицу $\Lambda$ считать разбитой на $k^{2}$ блоков размера $m \times m$. Матрица $I$ тоже должна представляться блочнодиагональной с $k$ единичными матрицами размера $m$ по диагонали. Вектор-столбцы $l_{i}^{\prime}, l_{i}^{\prime \prime} \in V_{n}^{*}$ и векторы $d_{i}^{\prime}, d_{i}^{\prime \prime} \in V_{n}, i=1, \ldots, r$, тоже разбиваются на $k$ подвекторов размерности $m$ каждый. Для удобства (хоть это и не обязательно) вектор-столбцы $L^{\prime}, L^{\prime \prime} \in V_{2 n}^{*}$ и векторы $D^{\prime}, D^{\prime \prime} \in V_{2 n}$ тоже можно считать разбитыми на $2 k$ подвекторов размерности $m$, причём один из подвекторов в $L^{\prime \prime}$ и $D^{\prime}$ обязательно ненулевой.

Матрица $C^{*}$ в условии (4) будет иметь похожий на рис. 1 вид, только матрица $\Lambda$ заменяется транспонированной матрицей $\Lambda^{T}$, верхние две строки переставляются с заменой $I$ на $\Lambda^{T}$, аналогично, правые два столбца переставляются с заменой $\Lambda$ на $I$. Правая верхняя клетка в матрице $C^{*}$ та же, что на рис. 1.

После линейных замен $\widehat{L^{\prime \prime}}=\left(\begin{array}{cc}0 & \Lambda \\ \Lambda & 0\end{array}\right) L^{\prime \prime}, \widehat{L^{\prime}}=\left(\begin{array}{cc}0 & \Lambda \\ \Lambda & 0\end{array}\right) L^{\prime}$ условие

примет вид

$$
\left({\widehat{L^{\prime \prime}}}^{T}, l_{r}^{\prime T}, \ldots, l_{1}^{T}\right) \widehat{C}^{*}=\left(l_{r}^{\prime \prime}, \ldots, l_{1}^{\prime \prime}, \widehat{L}^{\prime}{ }^{T}\right) .
$$

В условии (7) матрица $\widehat{C}^{*}$ имеет уже в точности представленный на рис. 1 вид, только матрица $\Lambda$ заменяется на $\Lambda^{T}$.

Пусть $Z$ - произвольная блочно-подстановочная матрица размера $n \times n$, соответствующая подстановке $\sigma \in S_{k}$, с невырожденными блоками $Z_{i}$ размера $m \times m$ на пересечении $i$-й полосы и $\sigma(i)$-й колонки, $i=1, \ldots, k$. На остальных местах в матрице $Z$ стоят нули. Из равенств (6) следуют равенства $B_{i+1} Z=\Pi_{i}\left(B_{i} Z \cdot Z^{-1}\right) Z \cdot Z^{-1} \Lambda Z+B_{i-1} Z, i=1, \ldots, r$.

При поиске линейных соотношений для схем Фейстеля операции шифрования (6) можно записывать (как это делали в конце п. 2) в новых координатах: $B_{i} Z=\tilde{B}_{i}, i=0,1, \ldots, k, k+1$. Тогда блоки $\Lambda^{T}$ в новой матрице $\widehat{\tilde{C}}^{*}$ заменятся на $\left(Z^{-1} \Lambda Z\right)^{T}$. Если $\left(Z^{-1} \Lambda Z\right)^{T}=\Lambda$ или

$$
Z^{-1} \Lambda Z=\Lambda^{T}
$$


то новая матрица $\widehat{\widetilde{C}}^{*}$ совпадёт с исходной матрицей $C$.

Сформулируем основное следствие представленных выше рассуждений, характеризующих идентичность применения линейного и разностного методов.

Теорема 1. Схема Фейстеля (6), у которой матрица $\Lambda \in G L(n, 2)$ удовлетворяет условию (8) при некоторой блочно подстановочной матрице $Z \in G L(n, 2)$, является самодвойственной.

Для матрицы $\Lambda$ циклического сдвига следующее из теоремы равенство максимальных чисел благоприятных номеров локальных подстановок в линейном и разностном методах было установлено А. А. Фроловым. В качестве $Z$ он использовал отображение $\Xi: V_{n} \rightarrow V_{n},\left(v_{1}, \ldots, v_{n}\right) \mapsto\left(v_{n}, \ldots, v_{1}\right)$.

Для $Z=\Xi$, кроме матриц циклических сдвигов, утверждению теоремы удовлетворяют все матрицы $\Lambda=\left\|\lambda_{i j}\right\|, i, j=1, \ldots, n$, симметричные относительно побочной диагонали: $\lambda_{i j}=\lambda_{n+1-j, n+1-i}, i, j=1, \ldots, n$, в частности, все циркулянтные матрицы. Утверждению теоремы, очевидно, удовлетворяют и все матрицы, симметричные относительно главной диагонали. В общем случае, когда $Z$ - произвольная блочно-подстановочная матрица, соответствующая подстановке $\sigma:\{1, \ldots, k\} \rightarrow\{1, \ldots, k\}$, условию (8) удовлетворяют все невырожденные двоичные матрицы $\Lambda=\left\|\Lambda_{i j}\right\|, i, j=1, \ldots, k$, с блоками $\Lambda_{i j}$ размера $m \times m$, для которых $\Lambda_{\sigma(j), \sigma(i)}^{T}=Z_{i}^{-1} \Lambda_{i j} Z_{j}, i, j=1, \ldots, k$.

6. Самодвойственные $\boldsymbol{X S L}$-сети. Наиболее распространённые $X S L$-сети блок открытого текста $a=B_{0} \in V_{n}$ преобразуют за $r$ итераций в блок шифрованного текста $b=B_{r} \in V_{n}$ в соответствии с равенствами

$$
B_{i}=\Pi_{i}\left(B_{i-1}\right) \cdot \Lambda, i=1, \ldots, r,
$$

где, как и в предыдущем пункте, $\Lambda \in G L(n, 2)$ - невырожденная двоичная матрица размера $n=m k, \Pi_{i}: V_{n} \rightarrow V_{n}-$ подстановка, $\Pi_{i}=\left(\pi_{i 1}, \ldots, \pi_{i k}\right)$, $\pi_{i j}: V_{m} \rightarrow V_{m}, \quad j=1, \ldots, k, \quad-$ локальные подстановки, зависящие от итерационных ключей, $V_{n}=\left(V_{m}\right)^{k}$.

Полагая вначале в качестве нелинейных преобразований не подстановки $\pi_{i j}, i=1, \ldots, r, j=1, \ldots, k$, а подстановки $\Pi_{i}, i=1, \ldots, r$, получим соотношения $x_{i}=B_{i-1}, y_{i}=\Pi_{i}\left(x_{i}\right)$. Матрица $C=[I, \Lambda, \ldots, \Lambda]$, аккумули- 
рующая в себе все линейные операции $X S L$-сети, имеет размер $(r+1) n \times(r+1) n$ и является блочно-диагональной с $r+1$ указанными блоками размера $n$ на диагонали. Если, как это и должно быть, нелинейными преобразованиями считать локальные подстановки $\pi_{i j}, i=1, \ldots, r$, $j=1, \ldots, k$, то матрица $C$ примет тот же вид. Нужно только, как и в случае схем Фейстеля, полагать блоки $I$ и $\Lambda$ разбитыми на $k^{2}$ подблоков размера $m \times m$ каждый.

Действуя по аналогии с п. 5, получаем: $C^{*}=\left[\Lambda^{T}, \ldots, \Lambda^{T}, I\right]$, а после замен $\widehat{L^{\prime \prime}}=\Lambda L^{\prime \prime}, \quad \widehat{L}^{\prime}=\Lambda L^{\prime}, \quad$ соответственно, $\widehat{C}^{*}=\left[I, \Lambda^{T}, \ldots, \Lambda^{T}\right]$. Далее, представляя рассматриваемое шифрпреобразование в виде

$$
a Z \cdot Z^{-1} \Pi_{1} Z \cdot Z^{-1} \Lambda Z \cdot Z^{-1} \Pi_{2} Z \cdot Z^{-1} \Lambda Z \cdot Z^{-1} \ldots Z \cdot Z^{-1} \Pi_{k} Z \cdot Z^{-1} \Lambda Z \cdot Z^{-1}=b
$$

для некоторой блочно-подстановочной матрицы $Z$ и полагая затем $a Z=\tilde{a}$, $\tilde{\Pi}_{i}=Z^{-1} \Pi_{i} Z, \quad \tilde{\Lambda}=Z^{-1} \Lambda Z, \quad b Z=\tilde{b}, \quad$ в новых переменных получим: $\widehat{\tilde{C}}^{*}=\left[I, \tilde{\Lambda}^{T}, \ldots, \tilde{\Lambda}^{T}\right]$. В результате, если выполнено условие (8), то $\widehat{\tilde{C}}^{*}=C$. Сказанное позволяет сформулировать аналог теоремы 1 для $X S L$-сетей.

Теорема 2. Шифрпреобразование (9), реализующее XSL-сеть с матрицей $\Lambda \in G L(n, 2)$, удовлетворяющей условию (8) при некоторой блочноподстановочной матрище $Z \in G L(n, 2)$, является самодвойственным.

Комментарии после теоремы 1 в определённой мере относятся и к теореме 2 .

В заключение автор выражает благодарность А.М. Зубкову за полезные критические замечания.

\section{Список литературы}

1. Biham E., Shamir A. Differential cryptoanalysis of DES-like cryptosystems // Proc. CRYPTO’90. Lect. Notes Comput. Sci. - 1991. - V. 537. - P. 2-21.

2. Matsui M. Linear cryptanalysis method for DES cipher // Proc. EUROCRYPT'93. Lect. Notes Comput. Sci. - 1994. - V. 765. - P. 386-397.

3. Matsui M. On correlation between the order of $S$-boxes and the strength of DES // Proc. EUROCRYPT'94. Lect. Notes Comput. Sci. - 1995. - V. 950. P. 366-375. 
4. Daemen J. Cipher and hash function design strategies based on linear and differential cryptanalysis. Doctoral Dissertation, K.U.Leuven. March, 1995.

5. Daemen J., Rijmen $V$. The Design of Rijndael: AES - The Advanced Encryption Standard. - Springer Verlag, 2002.

6. Rijmen $V$. Cryptanalysis and design of iterated block ciphers. Doctoral Dissertation. Katholieke Univ. Leuven. 1997. 